\title{
R-matrix calculation of low-energy electron collisions with $\mathrm{LiH}$
}

\author{
B. K. Antony ${ }^{\dagger, *}$, K. N. Joshipura*, N. J. Mason ${ }^{\dagger}$ and Jonathan \\ Tennyson $\ddagger$ \\ ${ }^{\dagger}$ Centre of Molecular and Optical Sciences, The Open University, Milton Keynes, UK \\ *Department of Physics, Sardar Patel University, Vallabh Vidyanagar, 388 120, \\ Gujarat, India \\ $\ddagger$ Department of Physics and Astronomy, University College London, Gower St., \\ London WC1E 6BT, UK
}

\begin{abstract}
Calculations are performed for electron scattering from LiH. These show that use of a closed-coupled expansion gives results significantly different from calculations performed at the static exchange level employed in all previous calculations. In particular the close coupled calculations find a Feshbach resonance which follows the first excited, a ${ }^{3} \Sigma^{-}$, state curve. This resonance could provide a route to dissociative attachment and electron impact vibrational excitation. Elastic scattering cross sections, which are very large, as well inelastic cross sections for excitation to the four lowest electronically excited states are presented as a function of $\mathrm{LiH}$ bondlength.
\end{abstract}

\section{Introduction}

Lithium hydride $(\mathrm{LiH})$ is the simplest stable metallic hydride. It is increasingly being studied as a potential storage agent for solid-state hydrogen storage with recent trials suggesting that it can exhibit the maximum reversible hydrogen capacity ever achieved in metal hydrides (almost $9 \mathrm{wt} . \%$ of $\mathrm{H}_{2}$ ). Hence $\mathrm{LiH}$ is believed to be likely to play a key role in the development of the 'hydrogen economy' with its benefits for hydrogen storage and transportation, hydrogen generation, hydrogen purification and the development of metal hydride compressors and hydrogen based heat pumps and refrigerators (McClaine et al. 2000). Indeed lithium hydride is already used as a nuclear reactor coolant and its adoption in the proposed ITER fusion reactor is under discussion.

Lithium hydride is readily made through the reaction of lithium metal with hydrogen gas to form the hydride, $\mathrm{LiH}$.

$$
2 \mathrm{Li}(\mathrm{s})+\mathrm{H}_{2}(\mathrm{~g}) \longrightarrow 2 \mathrm{LiH}(\mathrm{s})
$$

However lithium hydride is a rather unstable white/grey crystalline solid at room STP with a melting point of $689^{\circ} \mathrm{C}$ that is photolysed upon exposure to light, and reacts very violently with water, even the water vapour in the air. 
$\mathrm{LiH}$ (and its cation $\mathrm{LiH}^{+}$) is also believed to have played a crucial role in the early universe (Dalgarno \& Lepp 1987, Lepp et al. 2002, Bodo, Gianturco \& Martinazzo 2003). It has been proposed that spatial anisotropies in the cosmic radiation background can be produced by Thompson scattering of photons from simple molecules and/or electrons located in protoclouds (Dubrovich 1994). In addition the presence of molecules in the protogalatic clouds may induce small fluctuations in the Cosmic Ray Background (CRB) temperature when the population of ro-vibrational levels deviate from the Boltzmann equilibrium (Maoli et al. 1994). The efficiency of coupling between CRB photons and the primordial gas is dependent upon the chemical composition of the gas and therefore knowledge of the chemical composition of the early universe is necessary. Lithium hydride is of particular interest since its high dipole moment makes its rotational and ro-vibrational transitions particularly strong (Gianturco \& Giorgi 1996, Martinazzo et al. 2003, Bodo, Martinazzo \& Gianturco 2003).

One mechanism by which such ro-vibrational and dissociative transitions may be excited is electron impact, since at low energies these may be dominated by resonance formation. To date the only reported calculations on low-energy electron scattering from $\mathrm{LiH}$ are early ones designed to probe the dipolar effects in electron scattering calculations (Collins et al. 1980, Watson et al. 1981, Rescigno \& Orel 1981, Salvini et al. 1984). These works are based entirely upon using the so-called static exchange approximation. In this paper we report new estimates of the elastic and electronically inelastic scattering cross section at low energies using the R-Matrix method. These calculations show that the static exchange approximation does not give reliable results for low-energy collisions and, in particular, our calculations explore the formation of temporary bound states of $\mathrm{LiH}^{-}$. Such resonances are absent from the previous studies.

\section{Method}

The R-matrix method splits configuration space into an inner region, which is a sphere of radius $a$ about the target centre-of-mass, and an outer region. In this work values for $a$ were tested in the range $10-19 \mathrm{a}_{0}$, with the results presented below being for the largest value. Indeed, contrary to nearly all other closed shell diatomic systems we have studied, we were unable to obtain stable results for this system with $a<15 \mathrm{a}_{0}$.

In the inner region the total wavefunction for the system is written

$$
\Psi_{k}^{N+1}=A \sum_{I} \psi_{I}^{N}\left(x_{1}, \ldots, x_{N}\right) \sum_{j} \xi_{j}\left(x_{N+1}\right) a_{I j k}+\sum_{m} \chi_{m}\left(x_{1}, \ldots, x_{N}, x_{N+1}\right) b_{m k}(2)
$$

where $A$ is the anti-symmetrisation operator, $x_{n}$ is the spatial and spin coordinate of the $n^{\text {th }}$ electron, $\xi_{j}$ is a continuum orbital spin-coupled with the scattering electron and $a_{I j k}$ and $b_{m k}$ are variational coefficients determined in the calculation. The first summation runs over the target states used in the close-coupled expansion. The second summation runs over configurations $\chi_{m}$, where all electrons are placed in target molecular orbitals. The number of these configurations vary considerably with the model employed. 
With the wavefunction given by eq. (2), a static exchange calculation has a single Hartree-Fock target state in the first sum and the second sum runs over the minimal number of configurations, usually 3 or fewer, required to relax orthogonality constraints between the target molecular orbitals and the functions used to represent the configuration. Our fully close-coupled calculations used the five lowest target states, themselves represented by a configuration interaction (CI) expansion, in the first expansion and over a hundred configurations in the second. These configurations allow for both orthogonality relaxation and short-range polarisation effects. It should be noted that with CI target representations, the distinction between which configurations represent which of these effects becomes blurred.

$\mathrm{LiH}$ is a four electron system meaning that highly accurate electronic structure calculations are possible for this molecule (Cencek \& Rychlewski 2002, Cafiero \& Adamowicz 2002, Li \& Paldus 2003). The more limited experimental data has reviewd by Stwalley \& Zemke (1993).

In this work we followed Salvini et al. (1984) and used the Slater Type Orbital (STO) basis set of Cade \& Huo (1967). Wavefunctions for the first five target states of $\mathrm{LiH}, \mathrm{X}{ }^{1} \Sigma^{+}$, a ${ }^{3} \Sigma^{+}, \mathrm{A}{ }^{1} \Sigma^{+}, \mathrm{b}^{3} \Pi$ and $\mathrm{B}^{1} \Pi$, were constructed using orbitals generated from an self-consistent field (SCF) calculation. The orbitals were used for the static exchange calculation presented below. For the other calculations, CI target states were represented freezing the electrons in the $1 \sigma$ orbital and then allowing all configurations generated by distributing the other two electrons between the $2 \sigma, 3 \sigma, 4 \sigma, 1 \pi, 2 \pi$ and $1 \delta$ SCF orbitals.

Comparisons between the properties of our target states and those from previous studies are given in table 1 . It can be seen that our excitation energies are close to, but slightly above, those determined by more accurate calculations and, where available, by experiment. Potential curves for the geometries studied are given in Fig. 1.

$\mathrm{LiH}$ has a very large permanent dipole, of about $6 \mathrm{D}(1 \mathrm{D}=2.54$ a.u. $)$. It is important that for any scattering calculation that the target wavefunctions gives a reliable representation of this. As shown in Table 1, our results are close to those obtained previous studies, both experimental and theoretical.

Scattering calculations were performed using the UK molecular R-matrix program suite (Morgan et al. 1998). For diatomic targets these codes employ numerical basis functions in a partial wave expansion. Terms with $l$ up to 6 were explicitly retained in the partial wave expansion. Radial functions with energies up to $7 \mathrm{Ry}$ were also retained, higher functions were allowed for using a Buttle correction. For our largest calculations, those for which $a=19 \mathrm{a}_{0}$, this gave $103 \sigma$ and $87 \pi$ continuum functions. These continuum functions were Schmidt orthogonalised to the SCF orbitals used in the target CI. In the outer region, the R-matrices were propagated to $50.1 \mathrm{a}_{0}$ from where K-matrices were found using an asymptotic Gailitis expansion (Noble \& Nesbet 1984).

Calculations were performed for ${ }^{2} \Sigma$ and ${ }^{2} \Pi$ total symmetry and for ten LiH internuclear separations, those with $R=2.5,2.7,2.8,3.015,3.1,3.2,3.3,3.4,3.6,3.8$ and $4.0 \mathrm{a}_{0}$. The Born approximation was used to check for convergence of our cross sections 
Table 1. Properties of the LiH target, Top part: ground state energy and dipole moment in a.u.; lower part: excitation energies in $\mathrm{eV}$.

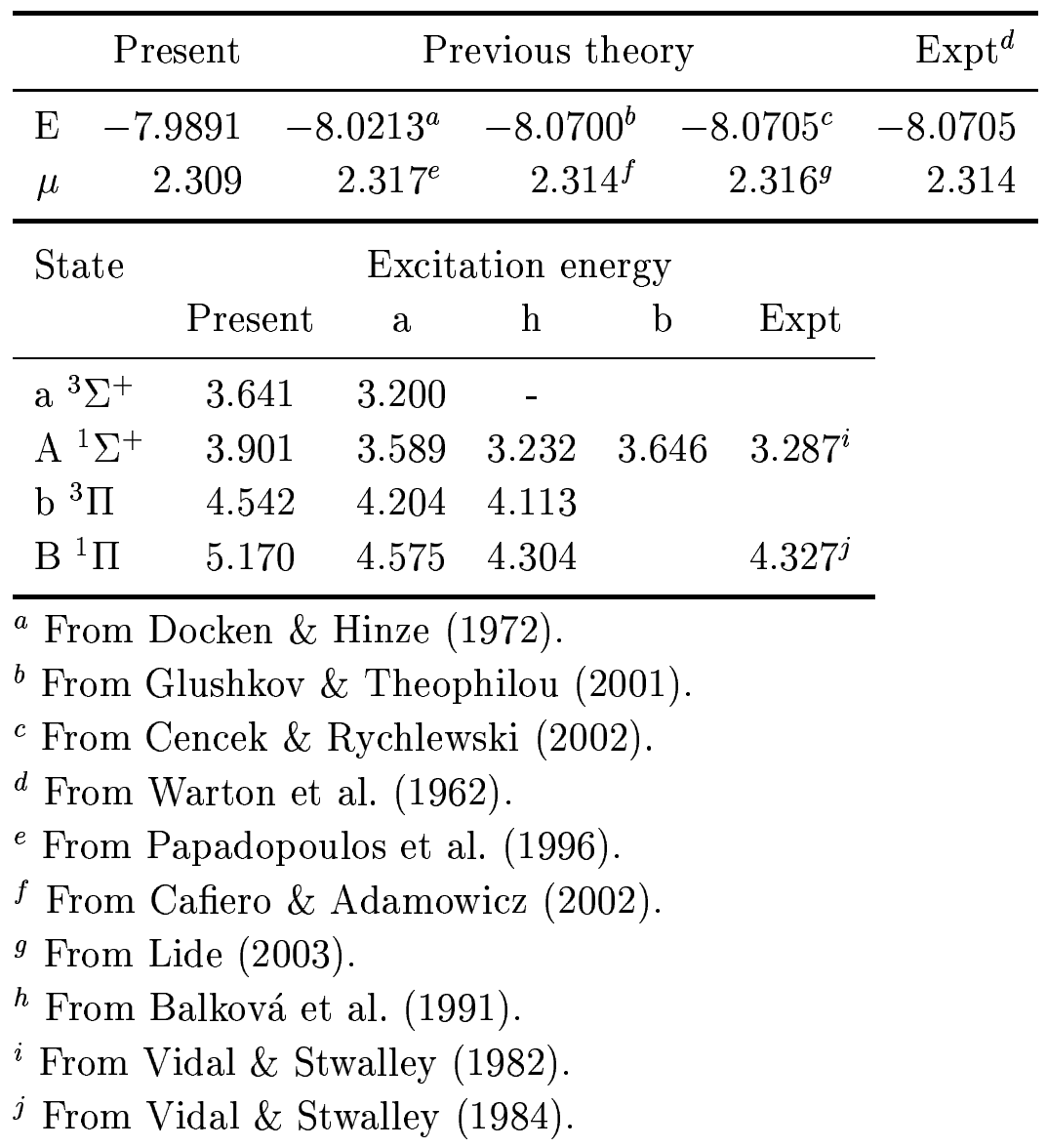

with respect to both the number of partial waves used in the basis set expansion and with total symmetries considered. As in previous studies of strong dipolar systems (Baluja et al. 2000, Rozum et al. 2003), the effects of rotational on the long-range interactions are corrected for at the same time using the adiabatic nuclei approximation. In these calculation the rotational constant and dipole moment were taken from the values given by our target CI calculation. For elastic scattering these corrections lead to a significant increase in the calculated cross sections whereas, even for dipole allowed transitions, the change in the electronic excitation cross sections was found to be negligible. Calculations were performed over a range of energies but the results reported below are largely restricted to electron collision energies below $6 \mathrm{eV}$. Above this energy there are open $\mathrm{LiH}$ target states which are not included in the calculation; in these circumstances artificial known features such as pseudo-resonances may result. 


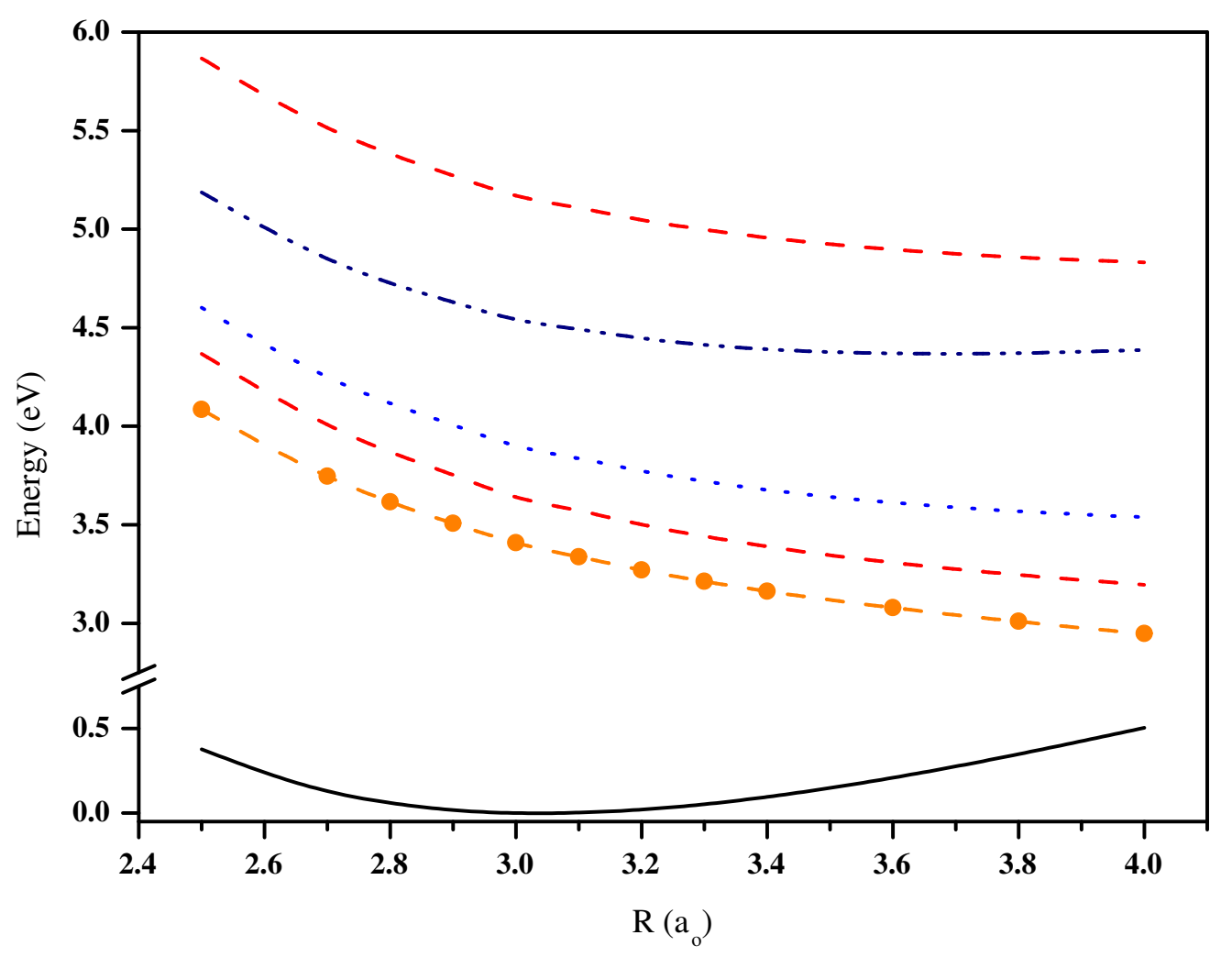

Figure 1. Calculated potential energy curves for LiH. The dot-dash curves gives the position of the ${ }^{2} \Sigma^{+}$resonance curve, the other curves from the bottom up are for the $\mathrm{X}^{1} \Sigma^{+}, \mathrm{a}^{3} \Sigma^{+}, \mathrm{A}{ }^{1} \Sigma^{+}, \mathrm{b}{ }^{3} \Pi$ and $\mathrm{B}{ }^{1} \Pi$ states. Energies are all relative to the minimum in the ground state curve.

\section{Results}

\subsection{Elastic scattering}

Our first calculations focused on comparing results obtained with a close-coupled (CC) expansion with those given by the static exchange (SE) approximation (figure 2 ). ${ }^{2} \Sigma$ eigenphase sums computed with the two models for LiH. At low collision energies, the two methods give similar eigenphases both increasing as the energy goes to zero. This is to be expected in a strongly dipolar system, however it should be noted that the two models differ in their representation of the $\mathrm{LiH}$ target ground state wavefunction.

As the scattering energy increases, the SE eigenphase decrease monotonically with energy, in line with the results from previous studies (Salvini et al. 1984). Our CC eigenphases are systematically higher than the SE ones and show considerable structure in the $3.5-4.5 \mathrm{eV}$ region which covers the threshold to excitation of the lowest two 


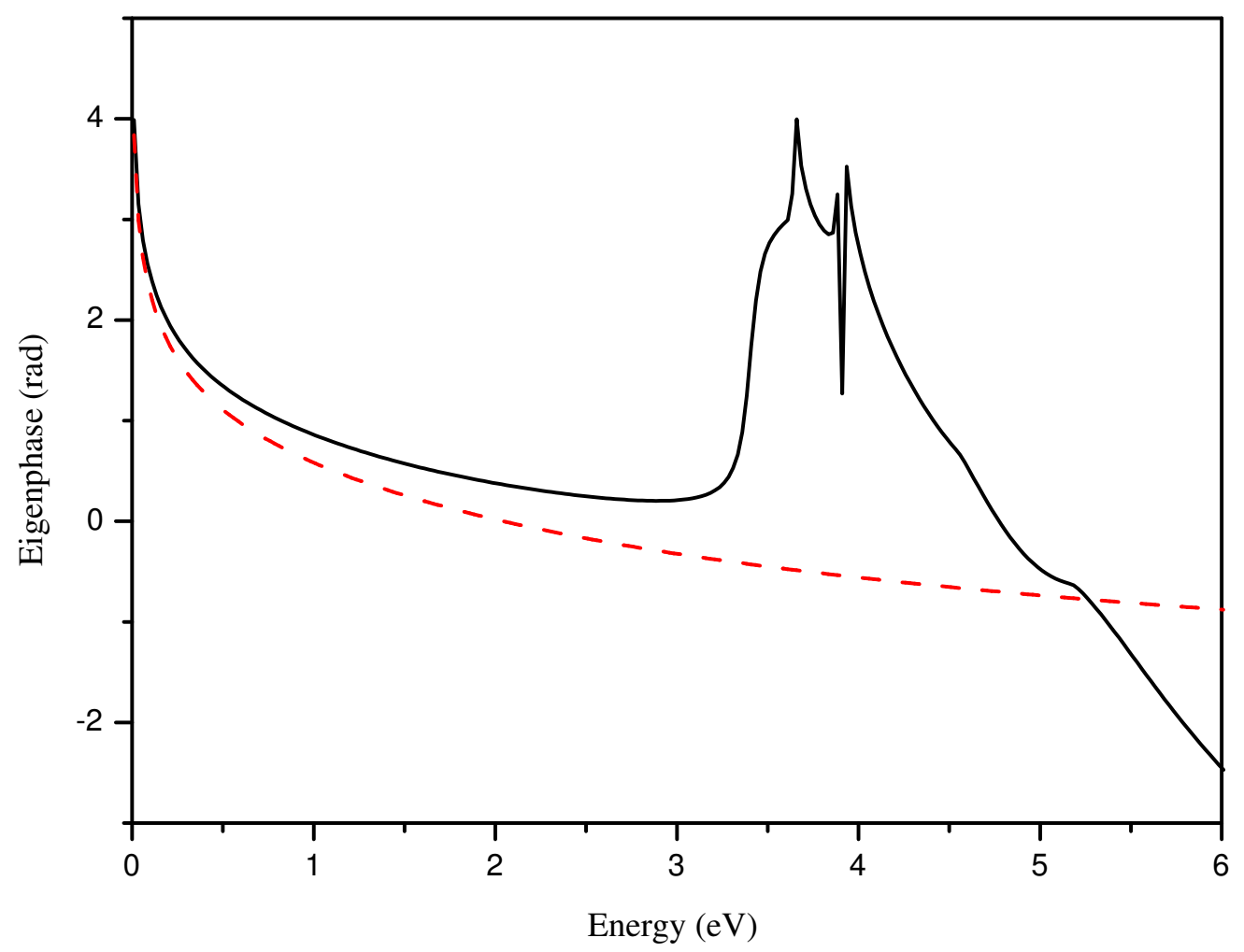

Figure 2. Electron $-\mathrm{LiH}$ eigenphases sums with ${ }^{2} \Sigma^{+}$symmetry computed for a fixed $\mathrm{LiH}$ bondlength of $R=3.015 \mathrm{a}_{0}$. The dashed curve is computed with static exchange (SE) approximation and the solid curve is computed using a close-coupled (CC) expansion. See text for details of these models.

electronic excited states. It would therefore appear that the SE approximation omits significant physical effects.

Analysis of the ${ }^{2} \Sigma \mathrm{CC}$ eigenphase sums show the clear signature of a resonance lying below the first electronic excited state, the a ${ }^{3} \Sigma^{+}$state. We fitted this resonance as a function of geometry using the resonance fitting program RESON (Tennyson \& Noble 1984). The resulting resonance curve is given in Figure 1, where it can be seen that this resonance follows closely the a ${ }^{3} \Sigma^{+}$state which is presumably the parent of this Feshbach resonance. Although it is well established that $\mathrm{LiH}^{-}$has a number of bound states (Gutsev et al. 1998), it would appear that no previous study has detected this resonance. As our calculation places the excited states of $\mathrm{LiH}$ at slightly higher energy than more elaborate calculations, we would expect our resonance curve to be high by an amount similar to that of our a ${ }^{3} \Sigma^{+}$state potential energy curve.

Figure 3 gives the variation of the resonance width as a function of internuclear separation. The resonance width is unusually constant over the range of geometries 


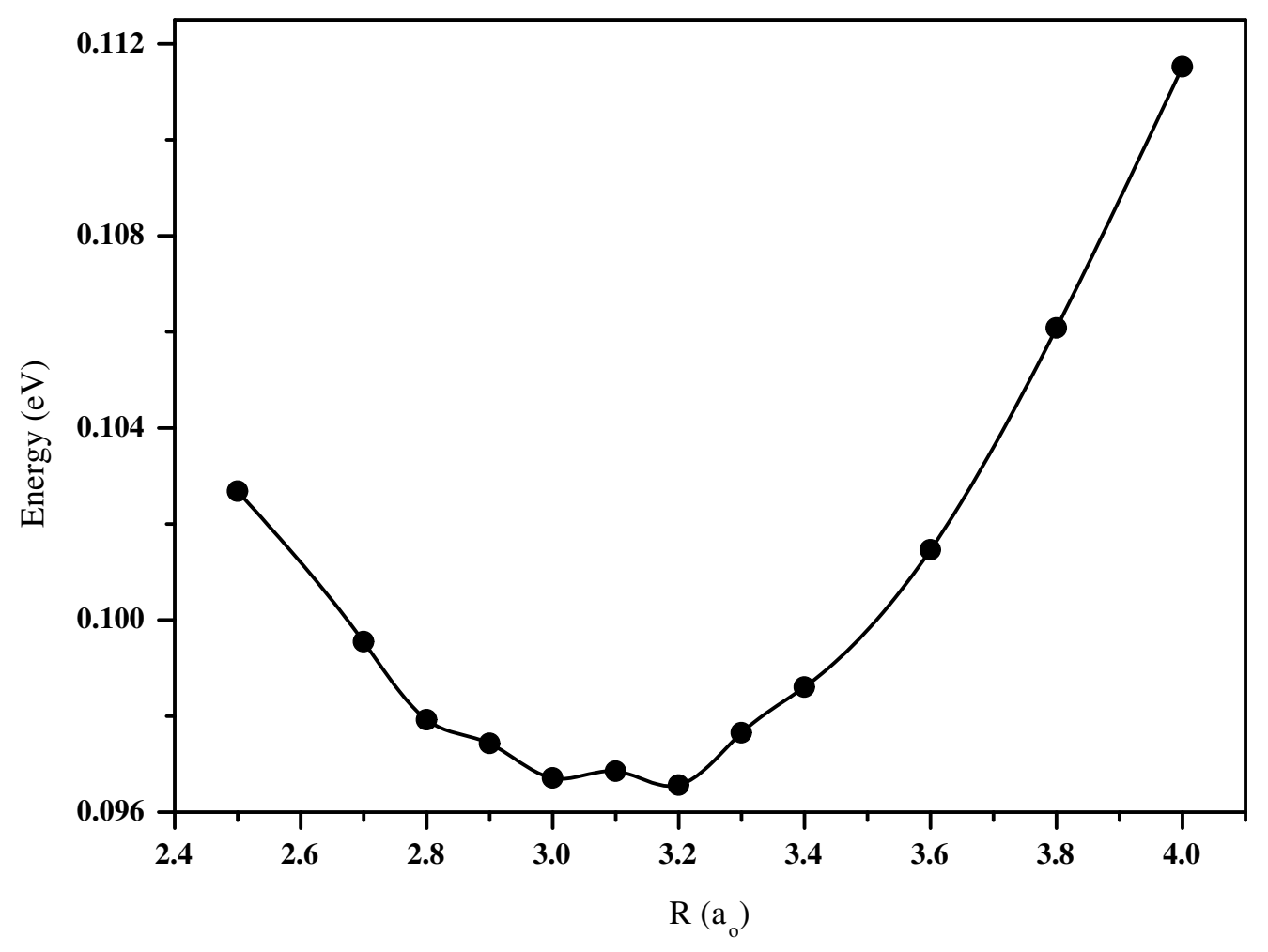

Figure 3. Width of the ${ }^{2} \Sigma^{+} \mathrm{LiH}^{-}$resonance as a function of $\mathrm{LiH}$ bond length.

considered (Rozum et al. 2003), varying only by about $10 \%$. It should be noted that the minor oscilations in the widths are almost certainly an artifact of the resonance fitting procedure and should be ignored.

Our ${ }^{2} \Pi$ symmetry calculations also show the clear signature of a resonance. However this resonance, which is somewhat broader having a width about $0.3 \mathrm{eV}$, lies at about $9 \mathrm{eV}$, well into the energy region where pseudo-resonances can occur. For this reason no detailed analysis of this resonance is presented.

Figure 4 presents our Born corrected elastic cross sections as a function of both electron scattering energy and $\mathrm{LiH}$ internuclear separation. For clarity only the curves for the equilibrium separation, $R_{e}=3.015 \mathrm{a}_{0}$ and the two extrema geometries we consider, $R=2.5$ and $4.0 \mathrm{a}_{0}$ are presented. As one would expect for scattering from a molecule with a large dipole moment, the cross sections are very large and are strongly peaked at zero. In contrast to less polar systems, the structures due to the resonances are almost negligible. For this reason we expect our results to be reliable up to the upper energy shown, $10 \mathrm{eV}$.

We should note that even the largest electronic excitation cross section, excitation to the $\mathrm{A}^{1} \Sigma^{+}$state, presented below is about three orders of magnitude smaller than 


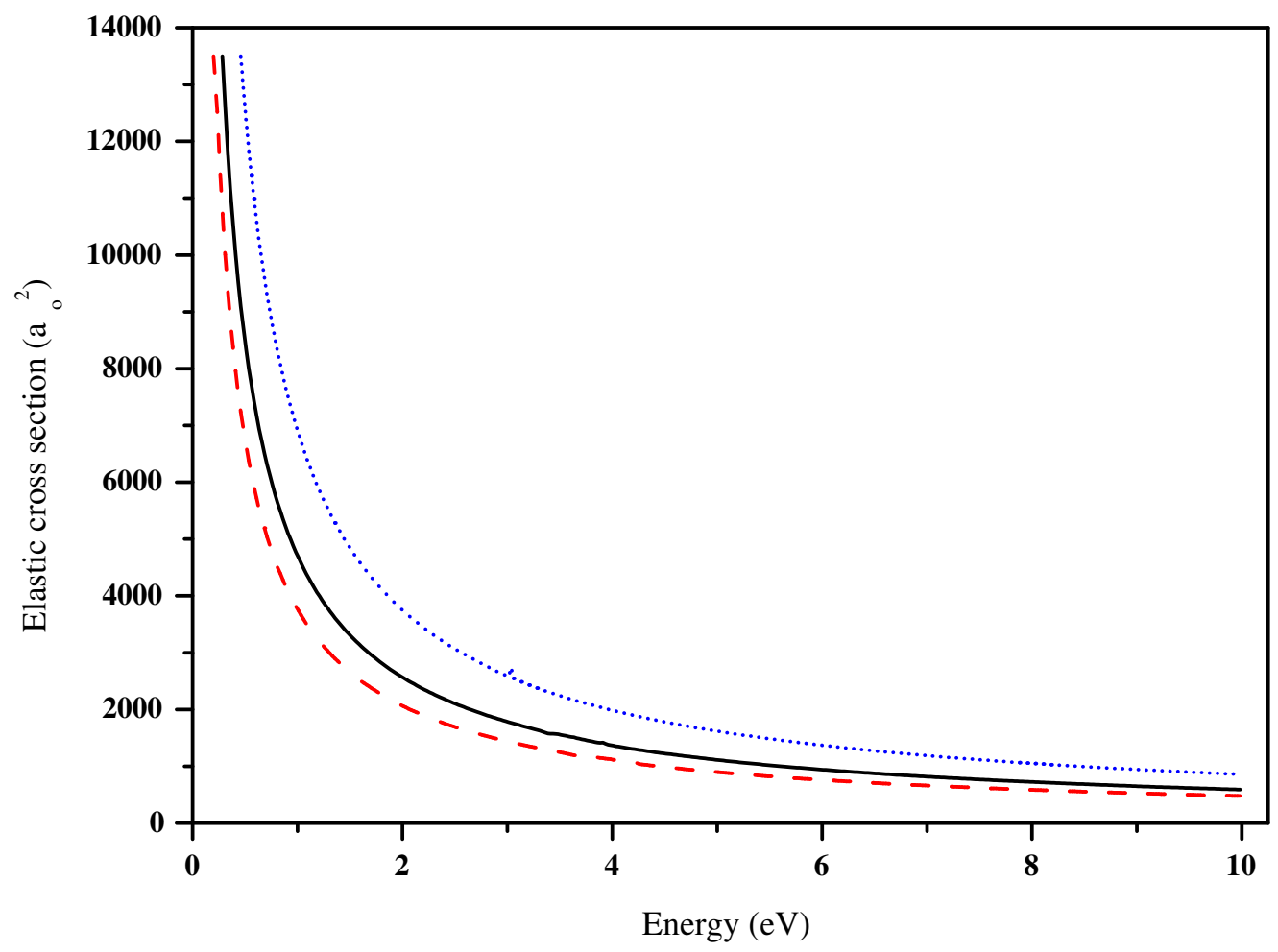

Figure 4. Elastic scattering cross section for three $\mathrm{LiH}$ bond lengths: dashed curve: $\mathrm{R}=2.5 \mathrm{a}_{0}$; solid curve: $\mathrm{R}=3.015 \mathrm{a}_{0}$; dotted curve: $\mathrm{R}=4.0 \mathrm{a}_{0}$.

the elastic cross section. This means that our elastic cross sections can be treated as total cross sections for all practical purposes.

\subsection{Electronic excitation}

Electron impact excitation cross sections were computed as a function of electron energy and $\mathrm{LiH}$ bondlength for the electronically excited states explicitly considered in the calculation. Results of these calculations are presented in Fig. 5.

The most notable feature of these cross sections is the pronounced feature nearthreshold which is present in $a^{3} \Sigma^{+}$and $\mathrm{A}^{1} \Sigma^{+}$excitation cross sections. In the case of excitation to the a ${ }^{3} \Sigma^{+}$state, this feature could be associated with the tail of the ${ }^{2} \Sigma^{+}$resonance discussed above. However this unlikely to be the case for the $\mathrm{A}^{1} \Sigma^{+}$ state. Analysis of the ${ }^{2} \Sigma^{+}$eigenphase sums, see Figure 2, show that there is a second pronounced feature which lies just above the a ${ }^{3} \Sigma^{+}$state. This feature, although fairly narrow, is truncated by the opening of the $\mathrm{A}^{1} \Sigma^{+}$state. This made it impossible to fit the feature with RESON although it is almost certainly also a Feshbach resonance. The near threshold structure in these cross sections is caused by this feature. No such 

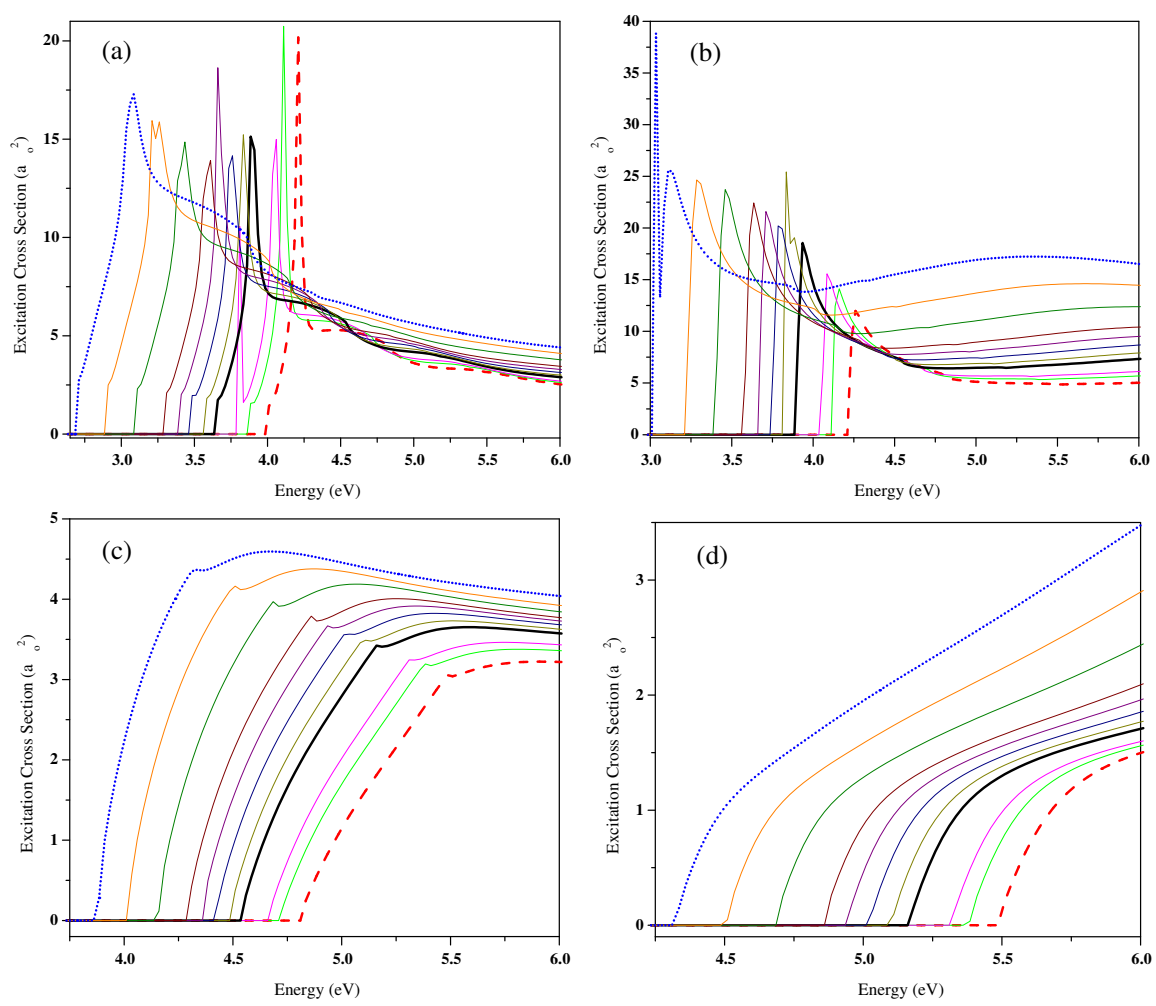

Figure 5. Electron impact excitation cross sections for excitation to (a) the a ${ }^{3} \Sigma^{+}$ state, (b) the $\mathrm{A}^{1} \Sigma^{+}$state, (c) the $\mathrm{b}{ }^{3} \Pi$ state and (d) the $\mathrm{B}{ }^{1} \Pi$ state. Each curve represents a different $\mathrm{LiH}$ bondlength, $R$. Starting from low energy the threshold appear in the order $R=4.0,3.8,3.6,3.4,3.3,3.2,3.1,3.015,2.8 .2 .7$ and $2.5 \mathrm{a}_{0}$.

feature is strongly visible in the $\mathrm{b}^{3} \Pi$ or $\mathrm{B}^{1} \Pi$ excitation cross sections.

\section{Conclusions}

We have performed a series of R-matrix calculations on low-energy electron collisions with lithium hydride. Sophisticated models which allow for coupling with low-lying excited electronic excited and other target polarisation effects give markedly different results from previous studies (Collins et al. 1980, Watson et al. 1981, Rescigno \& Orel 1981, Salvini et al. 1984) which used the static exchange approximation in which all polarisation effects are neglected. Given the dominance of the electron - dipole interaction for this system, which is correctly modelled at the static exchange level, this result confirms the observation that this model is not appropriate for treating low energy electron molecule collisions. It is such low energy, or near thermal, collisions which are 
important for most practical applications including those of $\mathrm{LiH}$, hydrogen storage and astrophysics, discussed in the introduction.

Our calculations identify a number of resonance features in the $\mathrm{LiH}^{-}$system which have not previously been noted. Particularly important is the lowest, ${ }^{2} \Sigma^{+}$ symmetry resonance. This resonance may lead to significant vibrational excitation. Furthermore, given the shape of this resonance curve, it will undoubtedly provide a route to dissociative attachment and hence provide a low energy route for destruction of $\mathrm{LiH}$ molecules by electrons. However extrapolating our resonance curve shows that it will only become bound at very large $\mathrm{LiH}$ internuclear separations meaning any quantitative description of this will have to take into account crossings with the very diffuse bound states of $\mathrm{LiH}^{-}$.

The present results may also have some interesting applications for the stability of $\mathrm{LiH}$ in the early universe and the appearance of any fluctuations in the Cosmic Ray Background. The consequences incorporation of the present results into astrochemical models should therefore be explored.

Finally the present calculations were performed fairly rapidly on standard desktop personal computers and it is therefore reasonable to ask if they can be extended higher collision energies. The ionisation potential of $\mathrm{LiH}$ is fairly low, $7.7 \mathrm{eV}$ (Lide 2003), and there are an infinite number of bound states converging to this point. Furthermore the higher-lying electronic states become increasingly diffuse and Rydberg-like which makes the constraint of keeping the target state within an R-matrix sphere of tractable size increasingly hard to satisfy. Very recently however Gorfinkiel \& Tennyson (2004) have developed a new R-matrix with pseudostates method which is capable of treating this intermediate energy region. So far this method has only been applied to collisions with the $\mathrm{H}_{3}^{+}$molecular ion, but it should provide a route to extending the range of collision energies considered in calculations such as these.

\section{Acknowledgements}

We wish to thank to Jimena Gorfinkiel and Iryna Rozum for useful discussions. B. K. Antony thanks the Association of Commonwealth Universities for a scholarship award in UK under which this work has been done. We also wish to acknowledge support for this work under the EU Framework V EPIC Network (Electron and Positron Induced Chemistry) HPRN-CT-2002-00179.

\section{References}

Balková A, Kucharski S A, Meissner L \& Bartlett R J 1991 J. Chem. phys. 95, 4311.

Baluja K L, Mason N J, Morgan L A \& Tennyson J 2000 J. Phys. B: At. Mol. Opt. Phys. 33, L677-L684. Bodo E, Gianturco F A \& Martinazzo R 2003 Phys. Rep. 384, 85-119.

Bodo E, Martinazzo R \& Gianturco F 2003 in A Miani, J Tennyson \& T van Mourik, eds, 'High Accuracy Potentials for Quantum Dynamics' CCP6, Daresbury p. 43.

Cade P E \& Huo W M 1967 J. Chem. Phys. 47, 614. 
Cafiero M \& Adamowicz L 2002 Phys. Rev. Lett. 88, 033002-1.

Cencek W \& Rychlewski J 2002 Chem. Phys. Lett. 320, 549.

Collins L A, Robb W D \& Morrison M A 1980 Phys Rev. A 21, 488-495.

Dalgarno A \& Lepp S 1987 in M. S Vardya \& S. P Tarafdar, eds, 'Astrochemistry' Vol. 118 of IAU symposium Reidel p. 109.

Docken K K \& Hinze J 1972 J. Chem. Phys. 57, 4928.

Dubrovich V K 1994 Astron. and Astrophys. Trans. 5, 57.

Gianturco F A \& Giorgi P G 1996 Phys Rev. A 54, 4073-4077.

Gorfinkiel J D \& Tennyson J 2004 to be published.

Glushkov V N \& Theophilou A K 2001 Phys. Rev. A 64, 064501.

Gutsev G L, Nooijen M \& Barlett R J 1998 Phys. Rev. A 57, 1646-1651.

Lepp S, Stancil P C \& Dalgarno A 2002 J. Phys. B: At. Mol. Opt. Phys. 35, R57-R80.

Li X Z \& Paldus J 2003 J. Chem. Phys. 119, 5346-5357.

Lide R 2003 CRC Handbook of Chemistry and Physics CRC Press LLC, Boca Raton FL.

Maoli R, Melchiorri F \& Tosti D 1994 ApJ 425, 372.

Martinazzo R, Tantardini G F, Bodo E \& Giantuco F A 2003 J. Chem. Phys. 119, 11241-11248.

McClaine A, Breault R W, Larsen C, Konduri R, Rolfe J, Becker F \& Miskolczy G 2000 Proceedings of 2000 US DOE Hydrogen Programme Review NREL/CP 570, 28890.

Morgan L A, Tennyson J \& Gillan C J 1998 Computer Phys. Comms. 114, 120-128.

Noble C J \& Nesbet R K 1984 Computer Phys. Comms. 33, 730-737.

Papadopoulos M G, Willets A, Handy N C \& Underhill A E 1996 Mol. Phys. 88, 1063.

Rescigno T N \& Orel A E 1981 Phys Rev. A 24, 1267-1271.

Rozum I, Mason N J \& Tennyson J 2003 J. Phys. B: At. Mol. Opt. Phys. 36, 2419-2432.

Salvini S, Burke P G \& Noble C J 1984 J. Phys. B: At. Mol. Phys. 17, 2549.

Stwalley W C \& Zemke W T 1993 J. Phys. Chem. Ref. Data 22, 87.

Tennyson J \& Noble C J 1984 Computer Phys. Comms. 33, 421-424.

Vidal C R \& Stwalley W C 1982 J. Chem. Phys. 77, 883.

Vidal C R \& Stwalley W C 1984 J. Chem. Phys. 80, 2697.

Warton L, Gold L P \& Klemperer W 1962 J. Chem. Phys. 37, 2149.

Watson D K, Rescigno T N \& McKoy B V 1981 J. Phys. B: At. Mol. Phys. 14, 1875-1882. 